\title{
Generation of atomic spin orientation with a linearly polarized beam in room-temperature alkali-metal vapor
}

\author{
P. Bevington $\odot,{ }^{1,2}$ R. Gartman $\odot,{ }^{1}$ and W. Chalupczak ${ }^{1}$ \\ ${ }^{1}$ National Physical Laboratory, Hampton Road, Teddington TW11 OLW, United Kingdom \\ ${ }^{2}$ Department of Physics, University of Strathclyde, Glasgow G4 ONG, United Kingdom
}

(Received 9 October 2019; revised manuscript received 14 January 2020; published 30 January 2020)

\begin{abstract}
Traditionally, atomic spin orientation is achieved by the transfer of angular momentum from polarized light to an atomic system. We demonstrate the mechanism of orientation generation in room-temperature caesium vapors that combines three elements: optical pumping, nonlinear spin dynamics, and spin-exchange collisions. Through the variation of the spin-exchange relaxation rate, the transition between an aligned and an oriented atomic sample is presented. The observation is performed by monitoring the atomic radio-frequency spectra. The measurement configuration discussed paves the way to simple and robust radio-frequency atomic magnetometers that are based on a single low-power laser diode that approach the performance of multilaser pump-probe systems.
\end{abstract}

DOI: 10.1103/PhysRevA.101.013436

\section{INTRODUCTION}

The generation of spin polarization is an essential step in the study and application of a large variety of systems, from solid-state samples [1] to cold atomic ensembles [2,3]. In the atomic physics domain, the standard method (optical pumping) relies on the transfer of angular momentum from polarized light to the atomic system [4]. While a typical scheme involves the interaction of an atomic sample with a circularly polarized laser beam propagating along a static magnetic field, other configurations, including different polarization [5-7] and number of lasers [8], have been demonstrated. Optical pumping also covers the transfer of optical angular momentum to the target atoms achieved via spin-exchange collisions (SEC) $[9,10]$. Another category of spin polarization processes combines optical pumping with nonlinear spin dynamics [11,12]. One particular realization of this is the so-called effect of alignment to orientation conversion, which involves the evolution of an atomic population imbalance in mutually orthogonal magnetic and electric fields [13-19]. In this way, tensor polarization (alignment), where the spins are aligned along a preferred axis but no preferred direction, can be transformed into a vector polarization (orientation), where spins are biased in one direction [18]. A similar effect has been achieved within the excited-state hyperfine sublevels in the presence of either an electric or a magnetic field [20-24].

In this paper, we explore the mechanism of the generation of spin orientation in room-temperature caesium vapor that combines three elements: (i) off-resonant optical pumping, (ii) nonlinear spin dynamics, and (iii) SEC (selective relaxation and coherence transfer [25-29]). (i) A linearly polarized laser beam moves the atomic population from the $F=3$ to the $F=4$ manifold through off-resonant optical pumping, while creating a population imbalance (alignment) within both levels [Fig. 1(a)]. The particular frequency detuning of the beam ensures that the majority of the population transferred to the $F=4$ level goes to either stretched state, i.e., sublevels with a maximum or minimum magnetic quantum number. While the dynamics within the $F=3$ level is defined by the resonant coupling to the laser field, the $F=4$ atomic spins evolve only in the presence of both weak far-off-resonant optical and SEC couplings. (ii) The weak coupling to the optical field drives the nonlinear spin dynamics that breaks the population distribution symmetry. In particular, it moves some of the population out of one of the stretched states, effectively making the atoms more prone to SEC relaxation (iii). As a consequence of these two factors (nonlinear spin dynamics and SEC), we observe suppression of components representing one of the spin directions that contributes to alignment and the generation of atomic orientation at low magnetic fields. In contrast to standard alignment to orientation conversion experiments [13-18], the change of the spin polarization discussed here is produced with parallel magnetic and electric (linearly polarized laser beam) fields. In a sense, the mechanisms demonstrated here are a generalization of the mechanism presented in Ref. [12], where the major difference between the two experiments is the initial state created by the pumping process. While the indirect pumping implemented in Ref. [12] creates a system with $80 \%$ of the population in the stretched state, here the starting point is an aligned state. This enables us to demonstrate a continuous transition from the aligned to oriented state with the scan of the Larmor frequency or the linearly polarized beam power.

The immediate implementation of the discussed technique is in the area of radio-frequency (rf) atomic magnetometry [30,31]. State-of-the-art rf atomic magnetometers take advantage of the properties of an oriented atomic polarization [30,32-34]. This polarization ensures immunity of the magnetometer signal to SEC decoherence [35-37] and enables a high signal-to-noise ratio (SNR) of the sensor. Possible applications of ultrasensitive rf magnetic field sensors cover a wide range of technologies from chemical analysis $[32,33,38]$ 
(a)

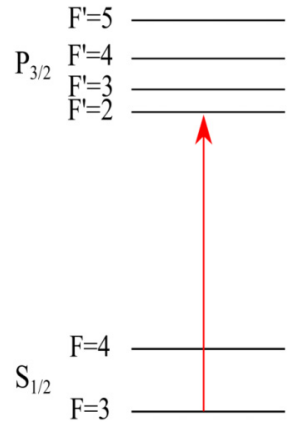

(b)

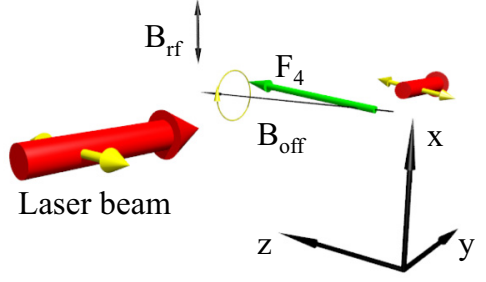

FIG. 1. (a) A linearly polarized laser beam, acting as the pump, transfers population between and creates a population imbalance within the $F=3$ and $F=4$ caesium ground-state manifolds, i.e., atomic spin along the direction of $B_{\text {off }}$, marked with a black arrow in panel (b). (b) A weak radio-frequency field, $B_{\text {rf }}$, orthogonal to the $\hat{z}$ axis creates coherences between adjacent $F=4$ Zeeman sublevels, causing the atomic spin polarization to precess around the $B_{\text {off }}$. The same linearly polarized laser beam, acting as the probe, monitors the spin precession via the Faraday effect. In some measurements, a weak circularly polarized beam propagating along the $\hat{z}$ axis is used to modify the population imbalance created by the linearly polarized beam.

to the nondestructive testing of materials [39-43]. A standard method for generating orientation in alkali-metal atoms (optical pumping) uses a circularly polarized laser beam operating on the D1 line [44]. For even more efficient pumping, an additional laser, the so-called repumper, is added to transfer the atoms from the other hyperfine ground state [8]. In this configuration, the signal readout is done by a linearly polarized laser beam operating on the D2 line. Consequently, the magnetometer arrangement involves two or three lasers operating at different frequencies. The concept discussed here achieves a comparable sensor performance (SNR) with a single laser diode, which significantly simplifies the instrumentation.

The following part contains a brief description of the experimental instrumentation. The components of the atomic spin orientation mechanism are explored through the dependencies of the rf spectroscopy signal on three measurement parameters (laser frequency detuning, beam power, and magnetic field strength), which are discussed in the subsequent sections.

\section{EXPERIMENTAL SETUP}

The measurements are performed in a shielded environment [12,29,34,37]. The ambient magnetic field is suppressed by the use of five layers of cylindrical shields with end caps, made from 2-mm-thick mu-metal. A solenoid inside the shield generates a well-controlled offset magnetic field, $B_{\text {off }}$, along the $\hat{z}$ axis [Fig. 1(b)], with a relative homogeneity exceeding $10^{-4}$ over the length of the cell. The atoms used are a caesium atomic vapor housed in a paraffin-coated, cross-shaped, quartz cell (22 $\mathrm{mm}$ in diameter and arm lengths of $32 \mathrm{~mm}$ ) at ambient temperature (atomic density $n_{\mathrm{Cs}}=0.33-1.0 \times 10^{11} \mathrm{~cm}^{-3}$ ). These atoms are optically pumped by a linearly polarized laser beam, $20 \mathrm{~mm}$ in diameter, propagating orthogonally to the direction of $B_{\text {off }}$ [Fig. 1(b)]. The polarization of the beam is parallel to $B_{\text {off }}$. The beam is provided by a distributed Bragg reflector diode laser operating on the caesium D2 line [Fig. 1(a)] and can be frequency stabilized within \pm 10 $\mathrm{GHz}$ with respect to the master laser frequency using offset locking. The same linearly polarized beam also acts as a probe of the spin precession via the Faraday effect [45], where the evolution of the collective atomic spin is mapped onto the polarization state of the linearly polarized probe beam $[8,30,34,44,46]$. The laser light transmitted through the cell is analyzed by a polarimeter consisting of a crystal polarizer oriented at $45^{\circ}$ with respect to the incident polarization and a commercial balanced photodetector. The two quadrature components of the resulting signal $X$ and $Y$ (where $X$ is the in-phase and $Y$ is the out-off-phase components, $R=$ $\sqrt{X^{2}+Y^{2}}$ ) are measured by a lock-in amplifier, referenced to the first harmonic of the driving rf field $\left(B_{\mathrm{rf}}\right)$ frequency.

\section{OFF-RESONANT PUMPING}

The population change, induced by off resonant pumping, in the $F=3$ and $F=4$ ground state can be described by a pair of rate equations [47]:

$$
\frac{d}{d t} G_{i}=G_{j} \sum_{F^{\prime}} R_{j, F^{\prime}} \beta_{j, F^{\prime}} \beta_{i, F^{\prime}}-G_{i} \sum_{F^{\prime}} R_{i, F^{\prime}} \beta_{i, F^{\prime}}\left(1-\beta_{i, F^{\prime}}\right),
$$

where index $F^{\prime}=2,3,4$, and 5 refers to the $6{ }^{2} P_{3 / 2}$ excitedstate levels, the pair of indices $(i, j)$ equal 3 and 4 and refer to the $F=3$ and $F=46^{2} \mathrm{~S}_{1 / 2}$ ground-state levels, and $\beta_{F, F^{\prime}}$ is the coupling coefficient between the ground state and the excited state. The absorption rate $R_{F, F^{\prime}}$ depends on the detuning of the pumping light from the relevant atomic transition. In our case, this dependence is dominated by Doppler broadening. Calculations based on the above equations show that the optimal condition (laser detuning) for population transfer from the $F=3$ to $F=4$ level and the generation of a population imbalance within the $F=4$ manifold are mutually exclusive. The former is optimized when the laser frequency is tuned in the vicinity of the $6{ }^{2} S_{1 / 2} F=3 \rightarrow 6{ }^{2} P_{3 / 2} F^{\prime}=4$ transition, at which the latter effect is minimized.

In the section below we experimentally identify the frequency range, which optimizes the buildup of orientation in the $F=4$ level.

Figure 2 shows the rf signal magnitude, $R$, as the frequency of the linearly polarized laser beam is scanned across the group of D2 line transitions involving the $F=3$ ground state (zero detuning represents the $6{ }^{2} S_{1 / 2} F=3 \rightarrow 6{ }^{2} P_{3 / 2}$ $F^{\prime}=2$ transition). The relatively small amplitude of $B_{\text {off }}$ (Larmor frequency $\sim 22 \mathrm{kHz}$ ) ensures that, on one hand, the contributions from both ground-state levels can be individually distinguished and, on the other hand, the Zeeman levels in a particular manifold are degenerate. The splitting between components of the $F=3$ spectral profiles is defined solely by the tensor light shift. In particular, as the laser frequency approaches the atomic resonance, the tensor light shift in $F=3$ increases and, consequently, so does the splitting between the components of the relevant profile. Due to the relatively large detuning from resonance, there is no significant splitting in the $F=4$ profile. Efficient pumping from the $F=3$ to the $F=4$ level, in the vicinity 


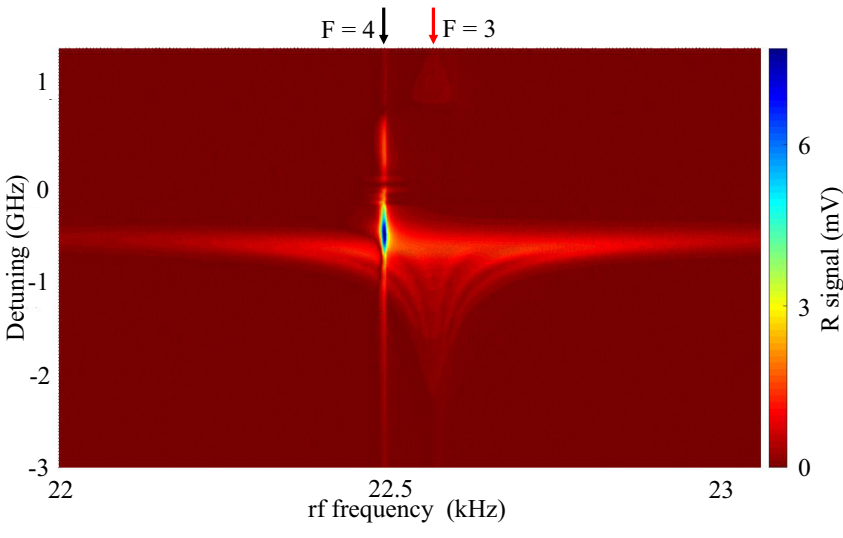

FIG. 2. Dependence of the rf signal amplitude, $R$, on the probe beam detuning from the $6{ }^{2} S_{1 / 2} F=3 \rightarrow 6{ }^{2} P_{3 / 2} F^{\prime}=2$ transition. The $F=3$ and $F=4$ resonances are marked with a red (gray) and a black arrow, respectively. The measurements have been made with a laser beam power of $5.9 \mathrm{~mW}$.

of the $6{ }^{2} S_{1 / 2} F=3 \rightarrow 6{ }^{2} P_{3 / 2} \quad F^{\prime}=4$ transition, results in the asymmetry in the $F=3$ signal amplitude with respect to the laser detuning. The exact detuning where the maximum in the $F=4$ signal is observed varies with the laser power and ranges from $\sim-416 \mathrm{MHz}$ (3.3 $\mathrm{mW})$ to $\sim-290 \mathrm{MHz}(10 \mathrm{~mW})$. While the observation of off-resonant $F=4$ pumping is similar to that observed on the D1 transition [48], there are two differences worth pointing out. First, the maximum pumping between the manifolds is reached for a nonzero laser detuning $(\sim-310 \mathrm{MHz}$ for the measurements represented in Fig. 2). Second, the character of the generated polarization within the $F=3$ (alignment) and $F=4$ (orientation) levels is different.

\section{NONLINEAR DYNAMICS}

The $F=4$ atomic spins only evolve in the presence of weak far-off resonant optical and SEC couplings. The linearly polarized light $\left(\vec{E}_{p}\right)$ couples to the atomic ground state through the tensor ac polarizability $\alpha_{2}$ (single-spin Hamiltonian, without the scalar part of the light shift $\sim \alpha_{2}\left(\vec{E}_{p} \cdot \hat{f}^{(i)}\right)^{2}$, where $\hat{f}^{(i)}$ is the total angular momentum operator of the $i$ th atom). Therefore, in general, the atomic spin dynamics will exhibit a nonlinear character $[11,12]$. The topic of atomic spin evolution in the presence of an electric and magnetic field has been the subject of a number of theoretical [19] and experimental $[11,12,16,17]$ studies. Mostly, the focus of these explorations was on measurement configurations where the two fields were orthogonal. In such a case, as shown in Ref. [19], the atomic polarization exposed to a static magnetic and an off-resonant ac electric field begins to oscillate between two types of atomic polarization with an oscillation period equal to $\frac{2 \pi}{\Delta \nu_{\mathrm{TLS}}}=$ $\frac{3}{2 \hbar(2 F-1)} \alpha_{2}\left|E_{p}\right|^{2}$, where $\Delta v_{\mathrm{TLS}}$ is equal to the line separation generated by the tensor light shift. As pointed out in Ref. [11] the nonlinear dynamics critically depends on the relative angle between the fields, $\theta$, i.e., in the arrangement discussed here, the angle between the laser beam polarization and the $\hat{z}$ axis. In particular, the nonlinear behavior, quantified in Ref. [11] by the spin decoherence, is twice as strong for an angle of
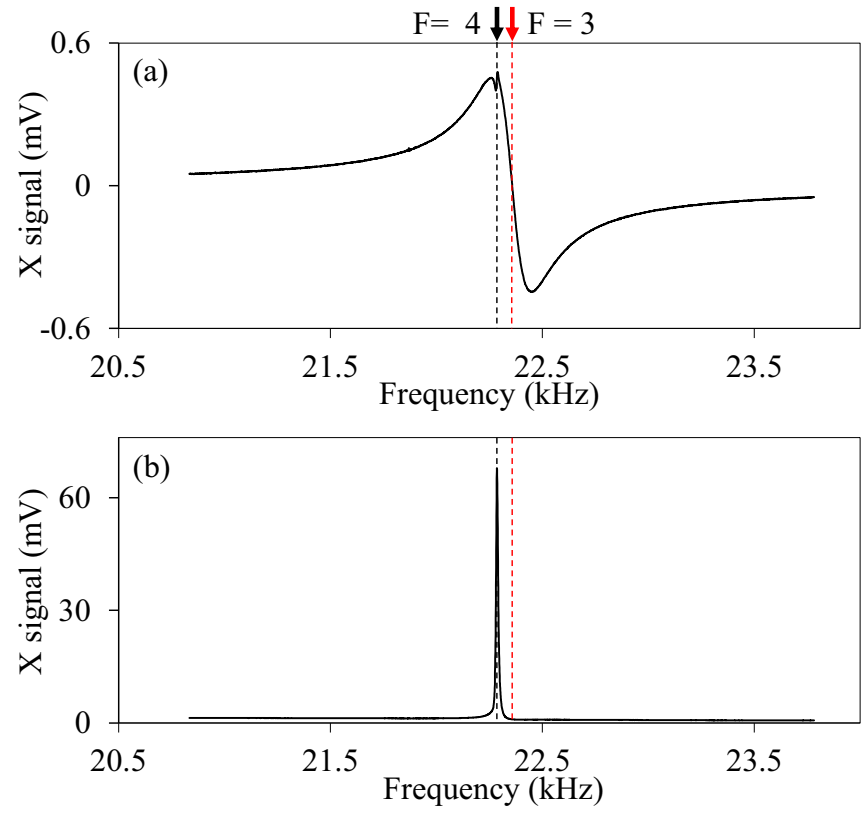

FIG. 3. The $X$ component of the $\mathrm{rf}$ spectroscopy signal recorded with the laser beam frequency tuned near the $6{ }^{2} S_{1 / 2} F=3 \rightarrow$ $6{ }^{2} P_{3 / 2} \quad F^{\prime}=2$ transition (detuning $-100 \mathrm{MHz}$ ). The $F=3$ and $F=4$ resonances are marked with a red (gray) and a black arrow, respectively. The transition from alignment (a) to orientation (b) can be seen in the spectral profile created by the $F=4$ coherences. The measurements were performed with a laser beam power of (a) $200 \mu \mathrm{W}$ and (b) $9.1 \mathrm{~mW}$.

$\theta=0^{\circ}$ than for $\theta=90^{\circ}$, while it disappears at $\theta=54^{\circ}$. Also, there has been a demonstration of an alkali-metal spin maser based on nonlinear spin dynamics, which was performed at $\theta=0^{\circ}[12]$. In the presence of SEC decoherence the behavior of the system is defined by a combination of processes, which is characterized by the ratio of the $\Delta v_{\mathrm{TLS}}$ and SEC relaxation rates. Calculation of the spectral splitting [49] indicates that $\Delta v_{\mathrm{TLS}}$ varies between 0.4 and $2 \mathrm{~Hz}$ for a linearly polarized beam power of 2 and $9 \mathrm{~mW}$, respectively. This is smaller than the relaxation rate due to SEC, $3 \mathrm{~Hz}$ [29]. This indicates that the SEC process dominates the spin dynamics, and while a small $\Delta v_{\mathrm{TLS}}$ can trigger nonlinear dynamics (similar to Ref. [12]), the system will not perform a full oscillation between atomic polarization states. Intuitively, the mechanism is similar to the evolution of the atomic spin in a near-zero static magnetic field, where the combination of three effects (optical pumping, slow spin precession around the magnetic field, and decoherence) effectively leads to a tilt of the steady-state spin polarization with respect to its initial state [50]. Here, the combination of (i) off-resonant pumping, which creates the alignment in the $F=4$ manifold, (ii) nonlinear spin dynamics that drives the alignment to orientation conversion, and (iii) SEC decoherence results in a change (asymmetry) in the initial population distribution. The effect of conversion from alignment to orientation is enhanced by the SEC coherence transfer, which is discussed in the following section.

Figure 3 shows the $X$ component of the rf spectra recorded with a laser power of $200 \mu \mathrm{W}$ [panel (a)] and $9.1 \mathrm{~mW}$ [panel (b)]. The positions of the $F=3$ and $F=4$ resonances are marked with red (gray) and black arrows, respectively. 

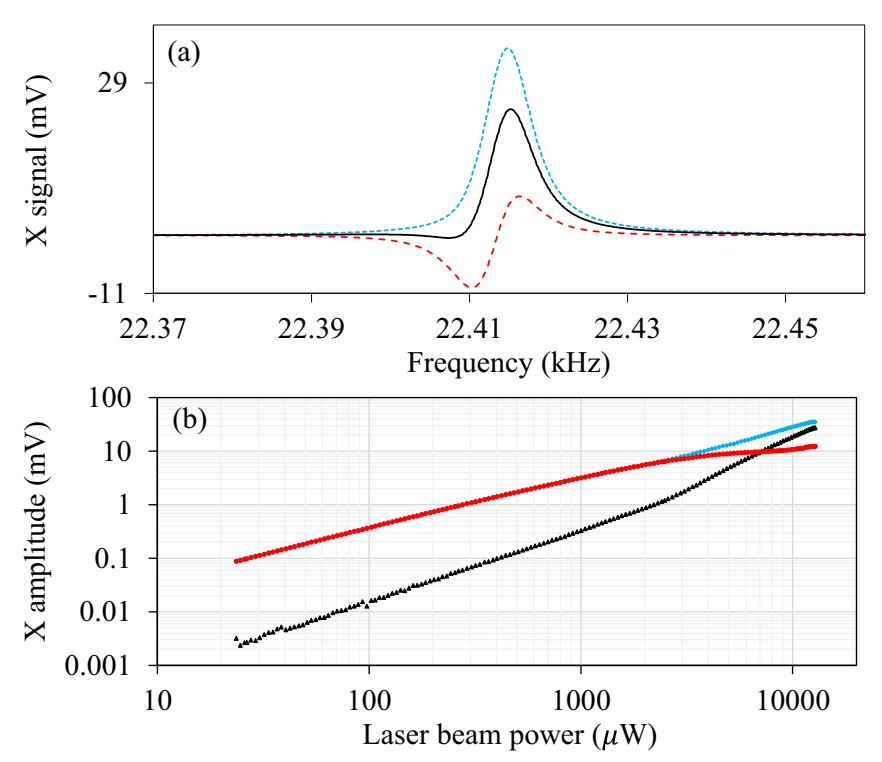

FIG. 4. (a) Magneto-optical-rotation signal, represented by the $X$ component of the rf spectroscopy signal, recorded with the linearly polarized beam only (solid black line), and in the presence of a circularly polarized pump beam with either orthogonal polarization component (dashed red line and dotted blue line). The linearly polarized beam power is $12.4 \mathrm{~mW}$ and the pump power is $17 \mu \mathrm{W}$. (b) Dependence of the $X$ component's amplitude on the laser beam power for the linearly polarized beam only (black triangles) and combined with a circularly polarized beam parallel to $B_{\text {off }}$ [red (gray) points and blue (light gray) diamonds represent the measurements with either of the two orthogonal circular polarizations for the pump beam].

Polarization-rotation resonances are observed when the rf field frequency matches the splitting between neighboring Zeeman sublevels introduced by $B_{\text {off }}$. In an aligned system, the rf response consists of two profiles with an opposite sign, leading to a dispersivelike lineshape. At low power [Fig. 3(a)], the rf spectrum consists of a large broad feature due to alignment in the $F=3$ level created by direct optical pumping, with a much smaller structure due to off-resonant excitation into the $F=4$ manifold. An increase in the laser beam power does not only translate into an increase of the $F=3$ and $F=4$ signal amplitudes [Fig. 3(b)]. While the character of the $F=3$ profile remains unaltered, the change in the symmetry of the $F=4$ signal indicates the presence of atomic orientation. The resonant coupling of laser light to the $F=3$ Zeeman sublevels results in power broadening of their corresponding spectral profiles, which contributes to the broad low-amplitude background visible in Fig. 3(b).

To confirm that the $F=4$ spectral profile represents atomic orientation, pumping with a circularly polarized (pump) beam propagating along the direction of $B_{\text {off }}(\hat{z}$ axis, Fig. 1) was added [37]. The pump beam is generated by a diode laser, frequency locked to the caesium $6{ }^{2} S_{1 / 2}$ $F=3 \rightarrow 6{ }^{2} P_{3 / 2} F^{\prime}=2,3$ crossover. The solid black line in Fig. 4(a) shows the rf spectrum for the $F=4$ profile recorded with only the linearly polarized beam. The dashed red (gray) and dotted blue (light gray) lines represent the case where one of the two orthogonal circular polarizations of the pump beam is added. The presence of the pump beam creates atomic orientation in the sample (parallel or antiparallel to $B_{\text {off }}$ ). If the orientation generated by the linearly polarized beam and the pump beam coincide, the amplitude of the observed profile increases [dotted blue (light gray) line in Fig. 4 (a)]. For the case with the opposite pump polarization, the signal amplitude decreases and the character of the spectrum changes [dashed red (gray) line]. The dependencies of the signal amplitude for the opposite pump beam polarizations are shown [red (gray) points and blue (light gray) diamonds] in Fig. 4(b). The amplitudes of the signals created by the orthogonally polarized pump beams are equal below $2 \mathrm{~mW}$ of probe power. The asymmetry in amplitudes above this power is produced by the sample orientation induced by the linearly polarized beam. The signature of this effect is also present in the amplitude data for the signal produced by only the linearly polarized beam (black triangles). The change from a linear to a quadratic slope in the amplitude power dependence, seen above $2 \mathrm{~mW}$, confirms the nonlinear character of the underlying mechanism.

\section{SPIN-EXCHANGE COLLISIONS}

The contribution of nonlinear spin dynamics to the generation of orientation is enhanced by SEC driven coherence transfer [25-29]. The effect relies on the macroscopic exchange of the excitation between different coherence modes subject to a resonance condition, which links the difference between the relevant coherence frequencies and the coherence relaxation rate [26]. If the size of the mismatch between the frequencies of the different modes is comparable to the SEC decoherence rate, rapid SECs average out the coherence precession with different Larmor frequencies leading to a prolonged oscillation at the averaged frequency [27]. Intuitively, the resonant condition for coherence transfer is equivalent to an overlap of the spectroscopic profiles of the relevant coherences in the rf spectrum. For the case considered here, the degeneracy between the $F=4$ Zeeman sublevel transition frequencies leads to an automatic realization of the resonance condition, i.e., the frequency mismatch (dephasing) between the precessing spins affected and not affected by SEC is negligible, so long as the SEC processes do not involve manifold change. Consequently, SEC processes do not contribute to relaxation, and a reduction in the SEC-dominated decoherence rate is observed [29]. One of the signatures of this coherence transfer is that the spectral profiles representing the relevant coherences group (merge) around the leading component of the spectrum [27,29].

Figure 5 shows the dependence of the two normalized quadratures ( $X$ and $Y$ ) of the rf spectroscopy signal on $B_{\text {off }}$ (in terms of Larmor frequency). The normalization takes into account the variation of the amplitude and the phase of the rf spectroscopy signal with operating frequency and was performed in the standard pump-probe configuration [37] over the same range of $B_{\text {off }}$. It is worth pointing out that SEC processes have a twofold contribution to the generation of orientation. Due to coherence transfer, tuning the strength of the offset magnetic field is equivalent to changing the value of the SEC relaxation rate. Reduction in the decoherence rate extends the duration of the nonlinear alignment to orientation evolution. Collisions between atoms in their 
(a)

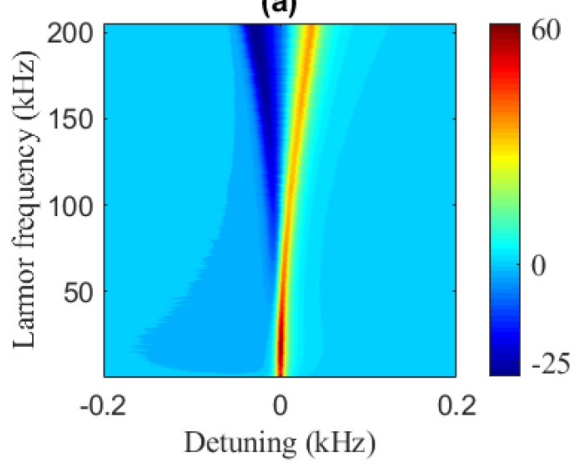

(d)

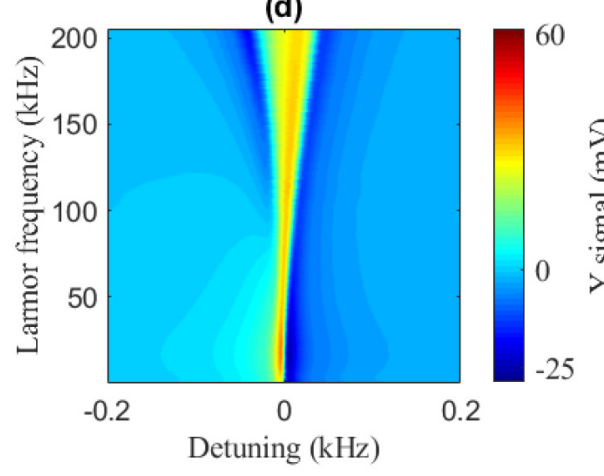

(b)

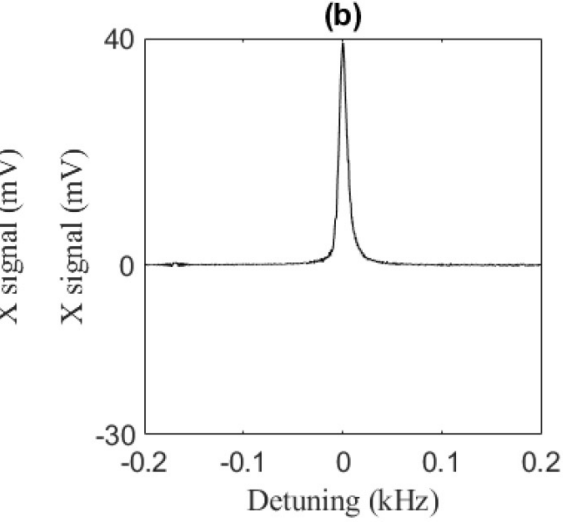

(e)

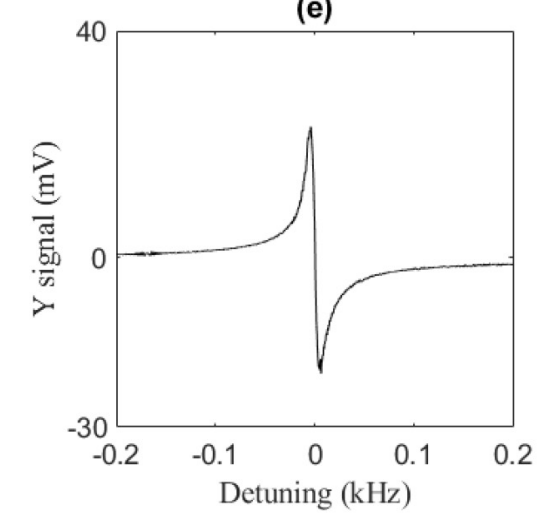

(c)

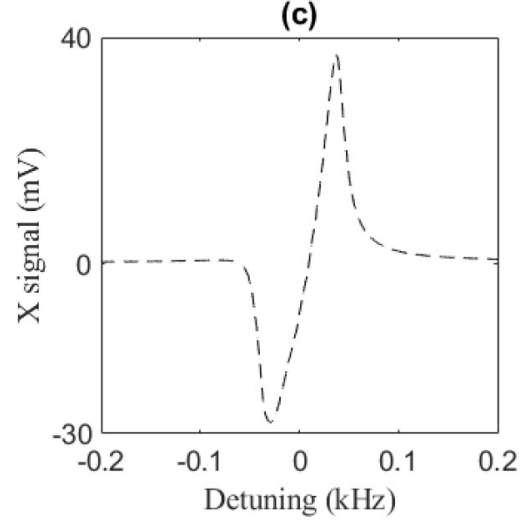

(f)

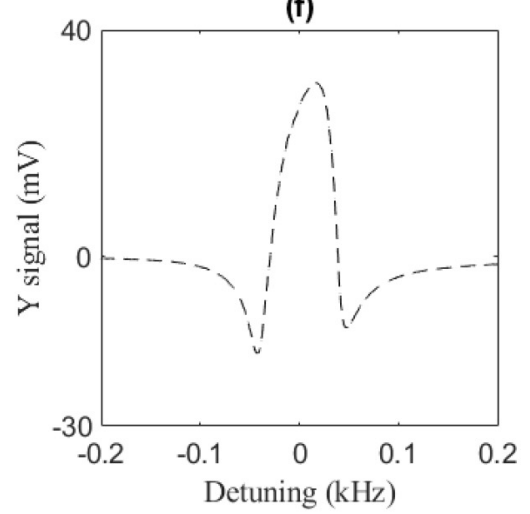

FIG. 5. Dependence of the $X$ (a) and $Y$ (d) quadrature components of the rf signal on the offset magnetic field strength (Larmor frequency). The frequency of the rf spectra is expressed in terms of the detuning from the center of the rf spectrum. Panels (b) and (e) are the $X$ and $Y$ components at a field strength equivalent to $0.6 \mathrm{kHz}$, while panels (c) and (f) are at $204.7 \mathrm{kHz}$. The measurements have been done with a laser beam power of $4.6 \mathrm{~mW}$.

stretched state does not introduce relaxation because the total angular momentum must be conserved; therefore selective SEC relaxation depopulates all but the stretched state. The spectral profile for large $B_{\text {off }}$ [Larmor frequency $\geqslant 100 \mathrm{kHz}$; Figs. 5(c) and 5(f)] has a shape typical for atomic alignment [Fig. 3(a)]. Decreasing $B_{\text {off }}$ increases the overlap of spectral components with an opposite sign, reducing the signal amplitude. Nonlinear spin dynamics results in a decrease in the population of the stretched state represented in Figs. 5(a) and $5(\mathrm{c})$ by the profile positioned at $\sim-0.05 \mathrm{kHz}$ detuning from resonance. This results in a higher relaxation rate for the coherences contributing to the part of the spectrum with a negative detuning. Consequently, the amplitude of the component with a negative detuning decreases more rapidly with $B_{\text {off }}$ than the other profile. The small frequency mismatch between these coherences (decreasing with the reduction of $B_{\text {off }}$ ) enhances coherence transfer and the buildup of atomic orientation observed over the frequency range below $20 \mathrm{kHz}$. The spectral profiles observed for Larmor frequencies $\leqslant 20 \mathrm{kHz}$ [Figs. 5(b) and 5(e)] represent the spin system in the oriented state.

The maximum signal-to-noise ratio, recorded in the measurement conditions discussed here, is 1.3-1.4 times lower than that measured for the optimized indirect pumping scheme [37]. Since the degree of population polarization described in Ref. [37], i.e., stretched state population as a fraction of total population, was found to be $80 \%$, we estimate the degree of polarization in the current configuration to be $72 \%$.

\section{CONCLUSIONS}

We have demonstrated the generation of atomic spin orientation in a room-temperature caesium vapor. The presence of atomic polarization is vital for the operation of a radiofrequency atomic magnetometer. The rf range of the presented technique $(1-30 \mathrm{kHz})$ is interesting in the context of magneticinduction-based nondestructive testing, where a low operating frequency translates into a deeper penetration depth of the (so-called primary) magnetic field [40-43]. The measurement configuration discussed here combines the efficient generation of the $F=4$ atomic orientation and off-resonant probing usually achieved with two or three independent lasers. The obvious benefit of the presented scheme is the simplicity of the instrumentation. Systematic measurements of the signalto-noise ratio (SNR) confirm that the discussed option delivers a SNR only 1.3-1.4 times lower than that recorded in the optimized pump-probe configuration [37]. The relatively sharp peak in the signal's frequency dependence (Fig. 2) allows for stabilization of the laser frequency despite strong saturation of the $F=3$ resonance. The difficulty that the orientation generated by the linearly polarized beam is observed over a relatively narrow range of $B_{\text {off }}$ could be overcome through the 
implementation of a degenerate pump-probe configuration, which involves a circularly polarized (pump along the $\hat{z}$ axis) beam and a linearly polarized (probe along the $\hat{y}$ axis) beam operating at the same frequency $\left(\sim 290 \mathrm{MHz}\right.$ from the $6{ }^{2} S_{1 / 2}$ $F=3 \rightarrow 6{ }^{2} P_{3 / 2} F^{\prime}=2$ transition). It is worth pointing out that this frequency for the pump beam is not far from that used in the optimized indirect pumping scheme, which means that a relatively small power, $\sim 100 \mu \mathrm{W}$, will be required [37]. The caesium ground-state hyperfine splitting $(9.172 \mathrm{GHz})$ defines the detuning of the laser frequency from $F=4$, which affects the signal amplitude and the nonlinearity strength. Hence, the use of ${ }^{85} \mathrm{Rb}$ vapor (hyperfine splitting of $3 \mathrm{GHz}$ ) would improve sensor performance in two ways. (i) Smaller detuning of the light monitoring the atomic spin evolution will increase the recorded signal amplitudes and potentially improve the SNR. (ii) The strength of the tensor couplings scales inversely with the square of the laser detuning [51]. Implementation of ${ }^{85} \mathrm{Rb}$ vapor would reduce the linearly polarized beam power threshold for nonlinear effects by nearly an order of magnitude $(\sim 200 \mu \mathrm{W})$. Preliminary tests indicate that the combination of a degenerate pump-probe and the use of ${ }^{85} \mathrm{Rb}$ vapor would enable efficient operation of the atomic magnetometer with $4 \mathrm{~mW}$ of laser light power, which is achievable from a single vertical-cavity surface-emitting laser diode.

\section{ACKNOWLEDGMENTS}

The work was funded by the UK Department for Business, Innovation and Skills as part of the National Measurement System Program. P.B. was supported by the Engineering and Physical Sciences Research Council (EPSRC) (Grant No. EP/P51066X/1).
[1] D. D. Awschalom, R. Hanson, J. Wrachtrup, and B. B. Zhou, Nat. Photonics 12, 516 (2018).

[2] A. Smith, B. E. Anderson, H. Sosa-Martinez, C. A. Riofrío, I. H. Deutsch, and P. S. Jessen, Phys. Rev. Lett. 111, 170502 (2013).

[3] T. Chalopin, C. Bouazza, A. Evrard, V. Makhalov, D. Dreon, J. Dalibard, L. A. Sidorenkov, and S. Nascimbene, Nat. Commun. 9, 4955 (2018).

[4] W. Happer, Rev. Mod. Phys. 44, 169 (1972).

[5] N. Fortson and B. Heckel, Phys. Rev. Lett. 59, 1281 (1987).

[6] W. M. Klipstein, S. K. Lamoreaux, and E. N. Fortson, Phys. Rev. Lett. 76, 2266 (1996).

[7] A. Andalkar, R. B. Warrington, M. V. Romalis, S. K. Lamoreaux, B. R. Heckel, and E. N. Fortson, Phys. Rev. A 65, 023407 (2002).

[8] W. Wasilewski, K. Jensen, H. Krauter, J. J. Renema, M. V. Balabas, and E. S. Polzik, Phys. Rev. Lett. 104, 133601 (2010).

[9] T. G. Walker and W. Happer, Rev. Mod. Phys. 69, 629 (1997).

[10] S. Appelt, A. B. Baranga, C. J. Erickson, M. Romalis, A. R. Young, and W. Happer, Phys. Rev. A 58, 1412 (1998).

[11] G. A. Smith, S. Chaudhury, A. Silberfarb, I. H. Deutsch, and P. S. Jessen, Phys. Rev. Lett. 93, 163602 (2004).

[12] W. Chalupczak and P. Josephs-Franks, Phys. Rev. Lett. 115, 033004 (2015).

[13] M. Lombardi, J. Phys. (Paris) 30, 631 (1969).

[14] C. Cohen-Tannoudji and J. Dupont-Roc, Opt. Commun. 1, 184 (1969).

[15] R. C. Hilborn, L. R. Hunter, K. Johnson, S. K. Peck, A. Spencer, and J. Watson, Phys. Rev. A 50, 2467 (1994).

[16] D. Budker, D. F. Kimball, S. M. Rochester, and V. V. Yashchuk, Phys. Rev. Lett. 85, 2088 (2000).

[17] M. C. Kuntz, R. C. Hilborn, and A. M. Spencer, Phys. Rev. A 65, 023411 (2002).

[18] M. Auzinsh, D. Budker, and S. M. Rochester, Optically Polarized Atoms (Oxford University, New York, 2010).

[19] S. M. Rochester, M. P. Ledbetter, T. Zigdon, A. D. WilsonGordon, and D. Budker, Phys. Rev. A 85, 022125 (2012).

[20] M. Kraińska-Miszczak, J. Phys. B: At. Mol. Phys. 12, 555 (1979).

[21] X. L. Han and G. W. Schinn, Phys. Rev. A 43, 266 (1991).

[22] J. Alnis and M. Auzinsh, Phys. Rev. A 63, 023407 (2001).
[23] M. Auzinsh, K. Blushs, R. Ferber, F. Gahbauer, A. Jarmola, and M. Tamanis, Phys. Rev. Lett. 97, 043002 (2006).

[24] M. Auzinsh, A. Berzins, R. Ferber, F. Gahbauer, L. Kalvans, A. Mozers, and A. Spiss, Phys. Rev. A 91, 053418 (2015).

[25] G. A. Ruff and T. R. Carver, Phys. Rev. Lett. 15, 282 (1965).

[26] S. Haroche and C. Cohen-Tannoudji, Phys. Rev. Lett. 24, 974 (1970).

[27] W. Happer and A. C. Tam, Phys. Rev. A 16, 1877 (1977).

[28] J. Skalla, G. Wackerle, and M. Mehring, Opt. Commun. 127, 31 (1996).

[29] W. Chalupczak, P. Josephs-Franks, B. Patton, and S. Pustelny, Phys. Rev. A 90, 042509 (2014).

[30] I. M. Savukov, S. J. Seltzer, M. V. Romalis, and K. L. Sauer, Phys. Rev. Lett. 95, 063004 (2005).

[31] W. Chalupczak, R. M. Godun, and S. Pustelny, Adv. At., Mol., Opt. Phys. 67, 297 (2018).

[32] S. K. Lee, K. L. Sauer, S. J. Seltzer, O. Alem, and M. V. Romalis, Appl. Phys. Lett. 89, 214106 (2006).

[33] I. M. Savukov, S. J. Seltzer, and M. V. Romalis, J. Magn. Res. 185, 214 (2007).

[34] W. Chalupczak, R. M. Godun, S. Pustelny, and W. Gawlik, Appl. Phys. Lett. 100, 242401 (2012).

[35] S. Appelt, A. B. A. Baranga, A. R. Young, and W. Happer, Phys. Rev. A 59, 2078 (1999).

[36] T. Scholtes, V. Schultze, R. IJsselsteijn, S. Woetzel, and H. G. Meyer, Phys. Rev. A 84, 043416 (2011).

[37] W. Chalupczak, R. M. Godun, P. Anielski, A. Wojciechowski, S. Pustelny, and W. Gawlik, Phys. Rev. A 85, 043402 (2012).

[38] G. Bevilacqua, V. Biancalana, Y. Dancheva, A. Vigilante, A. Donati, and C. Rossi, J. Phys. Chem. Lett. 8, 6176 (2017)

[39] A. Wickenbrock, S. Jurgilas, A. Dow, L. Marmugi, and F. Renzoni, Opt. Lett. 39, 6367 (2014).

[40] P. Bevington, R. Gartman, W. Chalupczak, C. Deans, L. Marmugi, and F. Renzoni, Appl. Phys. Lett. 113, 063503 (2018).

[41] P. Bevington, R. Gartman, and W. Chalupczak, Rev. Sci. Instrum. 90, 013103 (2019).

[42] P. Bevington, R. Gartman, and W. Chalupczak, J. Appl. Phys. 125, 094503 (2019).

[43] P. Bevington, R. Gartman, and W. Chalupczak, Appl. Phys. Lett. 115, 173502 (2019). 
[44] M. P. Ledbetter, I. M. Savukov, V. M. Acosta, D. Budker, and M. V. Romalis, Phys. Rev. A 77, 033408 (2008).

[45] Y. Takahashi, K. Honda, N. Tanaka, K. Toyoda, K. Ishikawa, and T. Yabuzaki, Phys. Rev. A 60, 4974 (1999).

[46] G. Bevilacqua, V. Biancalana, P. Chessa, and Y. Dancheva, Appl. Phys. B 122, 103 (2016)

[47] F. Atoneche and A. Kastberg, Eur. J. Phys. 38, 045703 (2017).

[48] W. Chalupczak, P. Josephs-Franks, S. Pustelny, and W. Gawlik, Phys. Rev. A 81, 013422 (2010).
[49] The calculated values of $\Delta v_{\mathrm{TLS}}$ are consistent with the broadening of the rf spectra that were the source of the amplitude data in Fig. 4(b) and those measured in W. Chalupczak, A. Wojciechowski, S. Pustelny, and W. Gawlik, Phys. Rev. A 82, 023417 (2010).

[50] I. K. Kominis, T. W. Kornack, J. C. Allred, and M. V. Romalis, Nature (London) 422, 596 (2003).

[51] J. Sherson, H. Krauter, R. K. Olsson, B. Julsgraard, and E. S. Polzik, J. Phys. B: At., Mol. Opt. Phys. 41, 223001 (2008). 